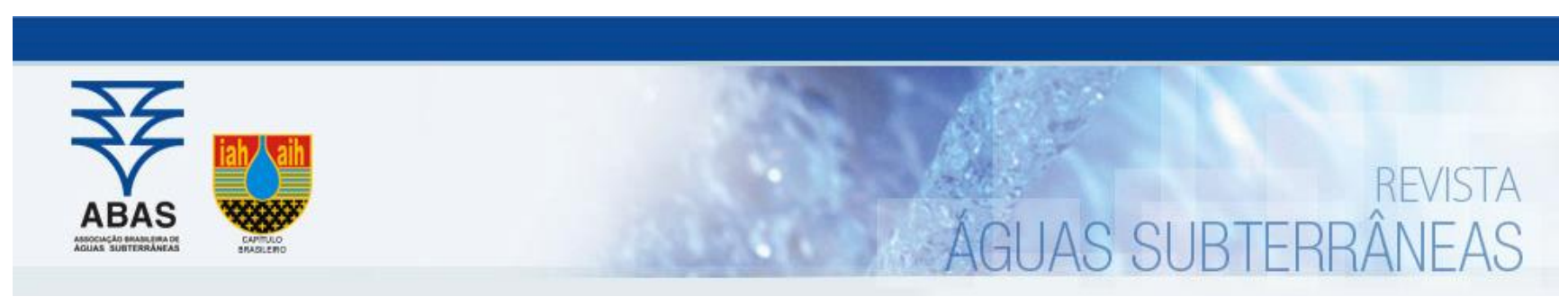

Artigos

\title{
Estimativa e Comparação do Potencial Hídrico Subterrâneo na Bacia Hidrográfica Taquari-Antas (RS)
}

\section{Estimation and Comparison of Groundwater Potential in Taquari-Antas Basin (RS)}

\author{
Tuane de Oliveira Dutra1; Pedro Antonio Roehe Reginato1; Talita Cabelera da Silva ${ }^{1}{ }^{\bowtie}$ \\ 1 Universidade Federal do Rio Grande do Sul (UFRGS), Porto Alegre, RS \\ $\triangle$ tuanehidrica@gmail.com, pedro.reginato@ufrgs.br, talita_c@hotmail.com
}

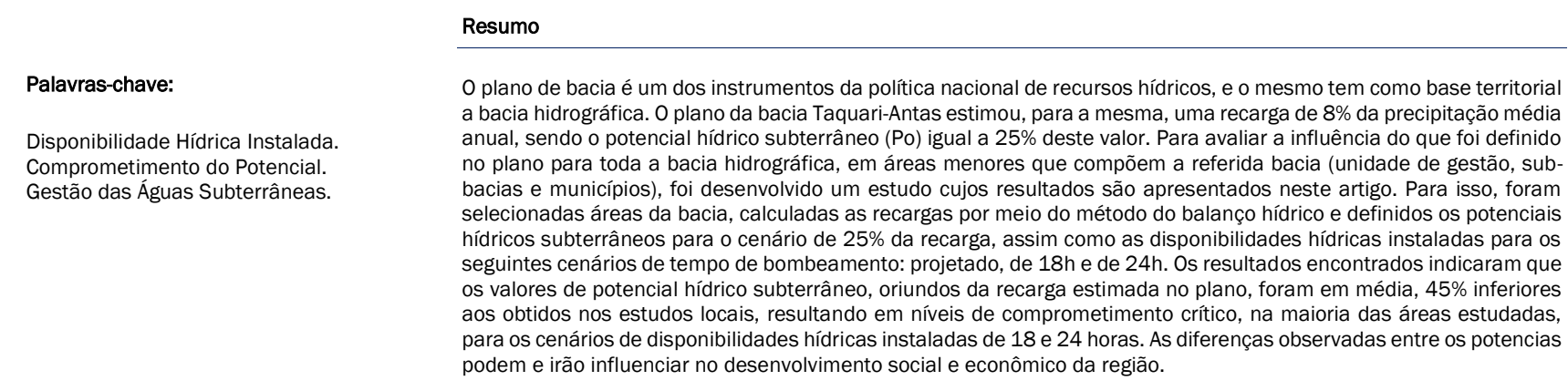

Keywords:

Installed Water Availability. Potential's Impairment.

Groundwater Management.

\section{Abstract}

Revisado por pares.

Recebido em: 23/01/2019.

Aprovado em: 18/03/2019.

\begin{abstract}
The basin plan is one of the instruments of the national water policy, and has the watershed as its territorial base. The Taquari-Antas basin plan estimated a recharge of $8 \%$ of the average annual precipitation for the same, with the groundwater potential (Po) equal to $25 \%$ of this value. To assess the influence of what has been defined in the basin plan for the entire watershed, in smaller areas that make up the referred basin (management unit, sub-basins and cities), a study was developed, whose results are presented in this article. For this, areas of the basin were selected, the recharge was calculated using the water balance method and the groundwater potential was defined for the $25 \%$ recharge scenario, as well as the installed water availabilities for the following scenarios of pumping time: projected, from $18 \mathrm{~h}$ and $24 \mathrm{~h}$. The results indicated that the values of groundwater potential, from the estimated recharge in the basin plan, were on average, $45 \%$ lower than those obtained in local studies, resulting in levels of critical impairment, in most of the areas studied, for the installed water availabilities scenarios of 18 and 24 hours. The observed differences among the groundwater potentials can and will influence the social and economic development of the region.
\end{abstract}

DOI: http:/dx.doi.org/10.14295/ras.v33i2.29476

\section{INTRODUÇÃO}

O plano de bacia, conforme BRASIL (1997), é um dos instrumentos da política nacional de recursos hídricos, tendo como área de atuação e de planejamento de gestão, a bacia hidrográfica. Nesses planos são realizados diversos estudos, os quais incluem análises de recarga, de reservas renováveis e de potencial hídrico subterrâneo, tais como os planos de bacia dos rios Taquari-Antas, Camaquã, Gravataí, Santa Maria, etc.

A recarga pode ser definida como o fluxo de água através da zona não saturada do solo, que alcança o nível freático e se adiciona ao reservatório de água subterrânea (HEALY, 2010). A recarga pode ocorrer naturalmente através da precipitação ou pela infiltração de rios, canais e lagos, ou também, pode ser induzida por atividades antrópicas como irrigação, urbanização e por recarga artificial através da criação de bacias de infiltração e poços de injeção direta (HEALY, 2010).

Conforme Costa (1998), a reserva renovável (Rr) é o volume de água acumulado no meio aquífero em função da porosidade eficaz, sendo variável anualmente em decorrência dos aportes sazonais de água superficial, do escoamento subterrâneo e dos exutórios. A recarga subterrânea contribui para aumentar a reserva subterrânea, renovável e permanente, de um aquífero (VASCONCELOS, 2005).

Segundo Todd (1959), o potencial hídrico subterrâneo é o vo- 
lume anual que pode ser explotado do aquífero sem que se produza um efeito indesejável, de qualquer ordem. Segundo Rebouças \& Fraga (1988), o potencial não é um valor fixo, podendo variar com: o desenvolvimento dos meios técnicos e financeiros da explotação, as mudanças no balanço hídrico e as condições econômicas do projeto. Ainda segundo o autor "o potencial hídrico subterrâneo de uma região é definido como a quantidade de água disponível para utilização, em forma de exploração racional"

Na bacia hidrográfica Taquari-Antas as águas subterrâneas representam a única fonte de recursos hídricos de cerca de $70 \%$ dos 119 municípios inseridos de forma parcial e integral na bacia (PLANO DE BACIA, 2011c). A bacia ocupa cerca de 9\% do território do Estado do Rio Grande do Sul, onde vivem cerca de 1.182 .000 habitantes, os quais representam $12 \%$ da população total do Estado, conforme o Plano de bacia (2011b), com base nos dados do IBGE (2010). Essa é uma região com alto índice de industrialização e áreas intensamente urbanizadas.

Em relação aos recursos hídricos subterrâneos na bacia, observa-se que 93,02\% dos recursos estão associados ao Sistema Aquífero Serra Geral (SASG),que conforme Machado et. al. (2005), é caracterizado por aquíferos fraturados associados às rochas vulcânicas da Formação Serra Geral. Segundo Reginato e Strieder (2004), as características hidrodinâmicas do SASG evidenciam um comportamento fortemente anisotrópico e, em função disso o potencial hídrico do aquífero é intensamente variado. Desta forma, se faz necessário estudos em escalas mais detalhadas, para uma estimativa mais realista desse parâmetro e suas variações no espaço.

Frente a este cenário, o presente estudo tem como objetivo avaliar e comparar, através de estudos locais e utilizando o valor de recarga calculado no Plano de Bacia (2011b), os volumes anuais do potencial hídrico em municípios, em sub-bacias e em unidades de gestão que compõem a bacia TaquariAntas. Além disso, procura-se avaliar a influência dessas diferentes escalas de análises nas estimativas do comprometimento dos recursos hídricos subterrâneos e sua implicação na gestão dos mesmos.

\section{MATERIAIS E MÉTODOS}

\subsection{Caracterização hidrogeológica}

O banco de dados construído foi elaborado a partir da coleta de informações realizada junto ao Sistema de Informações de Águas Subterrâneas (SIAGAS), Sistema de Outorga de Águas Subterrâneas do Rio Grande do Sul (SIOUT), Companhia Riograndense de Saneamento (CORSAN), Programa de Perfuração de Poços Artesianos (PAP/RS), prefeituras e empresas de perfuração. $O$ banco de dados em questão é constituído pelas seguintes informações: localização e quantidade de poços, vazão, tempo de bombeamento e tipo de uso dos recursos hídricos subterrâneos.

Análises estatísticas básicas foram aplicadas apenas para os dados de vazão e de tempo de bombeamento, porque esses são os dados dos poços utilizados para o cálculo da disponibilidade hídrica instalada. As análises envolveram medida de dispersão, neste caso, o desvio padrão, e medidas de tendência central: média e mediana.

\subsection{Seleção das áreas de estudo}

Foram selecionadas as áreas inseridas completamente no Sistema Aquífero Serra Geral, sendo considerados também os critérios mencionados a seguir:

- Sub-bacias e Unidades de Gestão: ser abastecida, na maior parte de sua área, por águas subterrâneas e ter uma quantidade de poços significativa (densidade superior a 0,2 poço/ $\mathrm{Km}^{2}$ );

- Municípios: ser abastecido exclusivamente por águas subterrâneas, e estar inserido completamente na área da sub-bacia.

O Plano de Bacia (2011a) dividiu a bacia Taquari-Antas em 7 unidades de gestão. As unidades que atenderam aos critérios estabelecidos acima foram as unidades de gestão UC e UG (Figura 1). O Plano de Bacia (2011a) também estabeleceu a delimitação das sub-bacias, sendo as selecionadas a Sub-bacia do Alto Rio Carreiro (SARC) e a Sub-bacia do Alto Rio Guaporé (SARG). Já os municípios que se enquadraram nos critérios foram Vila Maria e David Canabarro. 
Figura 1 - Mapa de localização das áreas de maior detalhe estudadas

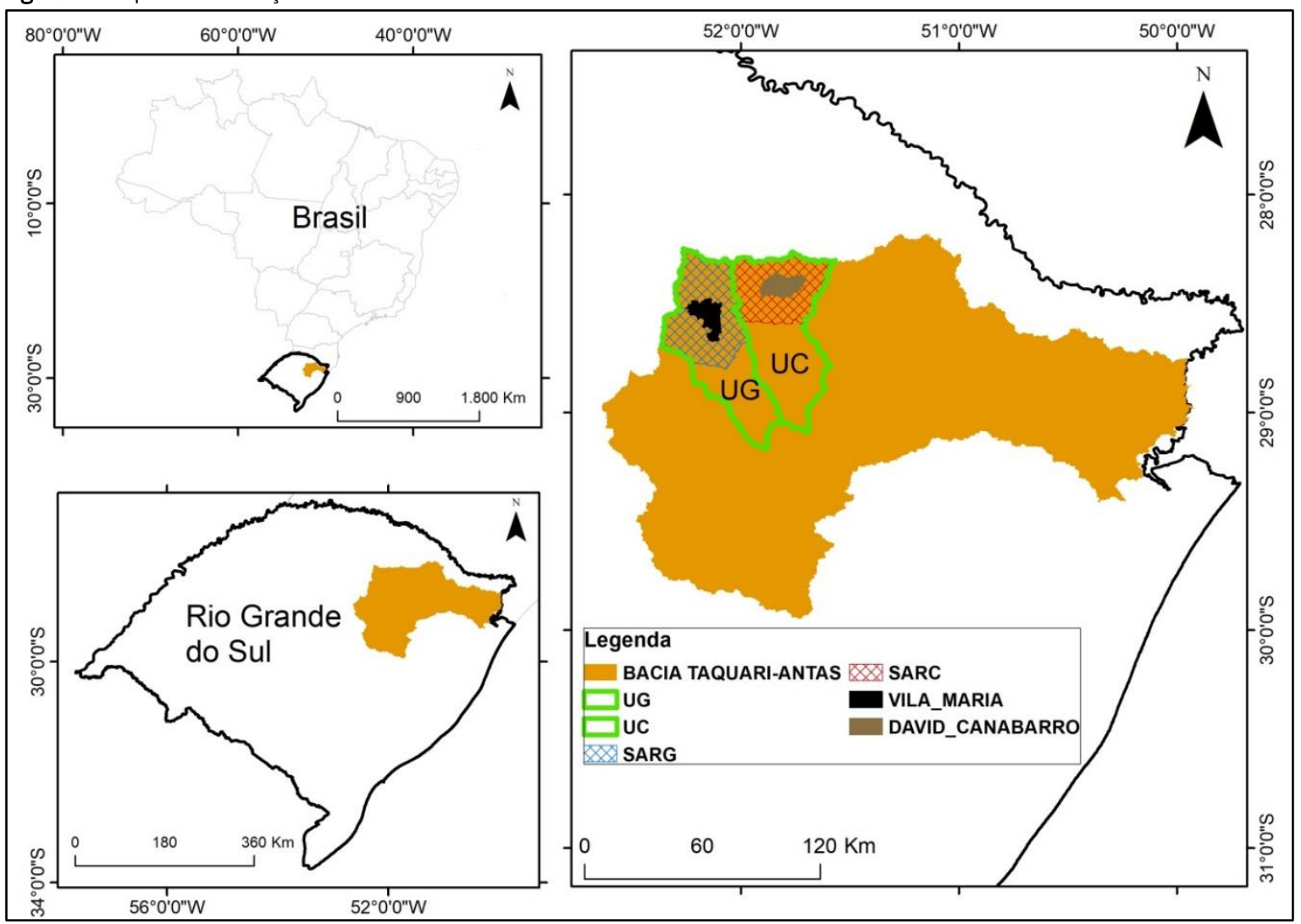

\subsection{Disponibilidade hídrica instalada total (Dit)}

A disponibilidade hídrica subterrânea é o volume de água passível de ser consumido através das captações de água já existentes (COSTA,1998).

Para esta etapa foram utilizados os dados obtidos no item 2.1 e o cálculo foi realizado para cada poço, sendo simulados três cenários, conforme as equações 1 , 2 e 3, considerando:

- o tempo de bombeamento como sendo de $24 \mathrm{~h}$ (Equação 1);

- o tempo de bombeamento cadastrado no projeto do poço (Equação 2);

- $\quad$ o tempo máximo permitido pelo Departamento de Recursos Hídricos $\quad$ (RS), de 18 h (Equação 3).

$$
\begin{aligned}
& \mathrm{Dit}_{24 \mathrm{~h}}=\Sigma(\mathrm{Q} .24 \mathrm{~h} .365) \\
& \mathrm{Dit}_{\mathrm{tbp}}=\Sigma(\mathrm{Q} . \mathrm{tbp} .365) \\
& \mathrm{Dit}_{18 \mathrm{~h}}=\Sigma(\mathrm{Q} .18 \mathrm{~h} .365)
\end{aligned}
$$

Onde:

Dit = Disponibilidade hídrica total $\left(\mathrm{m}^{3}\right)$;

$\mathrm{Q}=$ Vazão de projeto do poço $\left(\mathrm{m}^{3} / \mathrm{h}\right)$;

tbp $=$ Tempo de bombeamento projetado (h).

Nos casos dos poços que não possuíam dados de vazão foi utilizada a vazão mediana da área de estudo que o mesmo está inserido, devido aos valores elevados de desvio padrão observados em todas as escalas. Logo, por exemplo, para um poço sem informação inserido no município de Vila Maria, será considerado o valor mediano dos poços no município. O mesmo não ocorreu com os dados de tempo de bombeamento, sendo utilizado o tempo de bombeamento médio de cada escala para os poços sem essa informação.

Os valores totais de disponibilidade hídrica instalada (Dit), para cada área analisada, nos três cenários propostos, foram obtidos através da soma das disponibilidades hídricas instaladas individuais dos poços inseridos na área em questão.

Levando em consideração a parcela de poços não cadastrados na bacia, o Plano de Bacia (2011b), aplicou um percentual de $50 \%$ de aumento sobre o valor da Dit calculada, para que a mesma se aproximasse de volumes mais realistas. Sendo assim, neste estudo também será admitido o valor de $50 \%$ sobre o valor total calculado, para a estimativa daDit nas áreas estudadas.

\subsection{Potencial hídrico subterrâneo}

O potencial hídrico subterrâneo (Po) foi calculado para as diferentes áreas estudadas, conforme a equação 4, considerando $25 \%$ das reservas renováveis (Rr). A porcentagem aplicada sobre a Rr foi a mesma utilizada no Plano de Bacia (2011b), calculada conforme equação 5.

$\mathrm{Po}=(0,25 \cdot \operatorname{Rr}) \cdot \mathrm{A}$

Onde: 
Po = Potencial hídrico subterrâneo $\left(\mathrm{m}^{3}\right)$;

$\mathrm{Rr}=$ Reserva renovável $(\mathrm{m})$;

$A=$ Área estudada $\left(m^{2}\right)$.

$\operatorname{Rr}=(\operatorname{R} . P) / 1000$

Onde:

$\mathrm{Rr}=$ Reserva renovável $(\mathrm{m})$;

$\mathrm{R}=$ Recarga (\%);

$\mathrm{P}=$ Precipitação média da área em questão $(\mathrm{mm})$.

No Plano de Bacia (2011b), foi prevista uma recarga igual a $8 \%$ da precipitação média anual, sendo que esse percentual foi adotado para o cálculo da reserva renovável do plano (Rrp), equação 6 . Ao aplicar o percentual de $25 \%$ sobre a Rrp foram obtidos os volumes de potencial hídrico subterrâneo do plano (Pop), conforme a equação 7 .

$\operatorname{Rrp}=(0,08 \cdot P) / 1000$

Onde:

Rrp = Reserva renovável obtida com a estimativa do plano (m);

Pop $=(0,25 \cdot \operatorname{Rrp}) . A$
Onde:

Pop = Potencial hídrico subterrâneo estimado com a recarga calculada no plano de bacia $\left(\mathrm{m}^{3}\right)$.

A estimativa da recarga foi realizada por meio da aplicação do método do balanço hídrico (Equação 8), com o objetivo de comparar os valores obtidos com os definidos no plano e analisar a influência dos mesmos nas estimativas do cálculo do potencial das diferentes áreas de estudo (unidades de gestão, sub-bacias e municípios).

$R=P-E T R-R_{\text {off }}$

Onde:

$\mathrm{R}=$ Recarga $(\mathrm{mm})$;

$\mathrm{P}=$ Precipitação $(\mathrm{mm})$;

ETR = Evapotranspiração real $(\mathrm{mm})$;

$R_{\text {off }}=$ Escoamento superficial $(\mathrm{mm})$.

A precipitação média anual foi obtida através de 14 estações pluviométricas do Hidroweb (Figura 2), já previamente cadastradas e consistidas no Plano de Bacia (2011b), as quais possuem no mínimo 15 anos de observação, com dados que representam a situação climática atual. Foram utilizadas as seguintes estações para o cálculo da precipitação média anual em cada área de estudo:

Figura 2 - Distribuição espacial das estações pluviométricas utilizadas

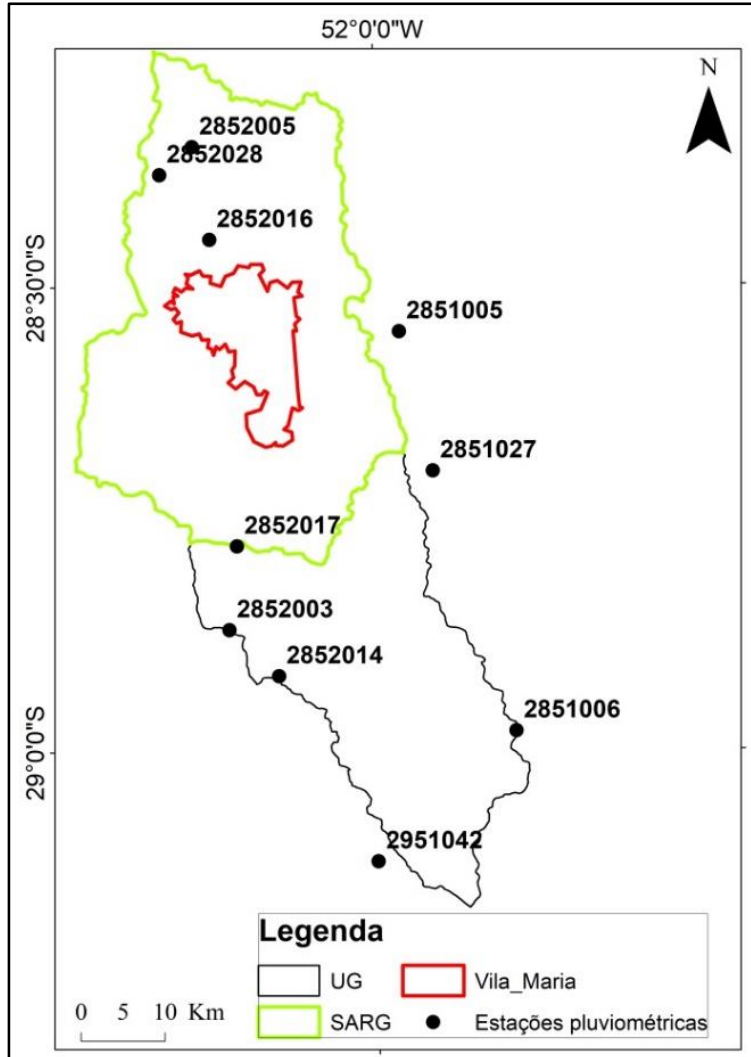

O mapa de evapotranspiração foi realizado através dos dados de evapotranspiração potencial do Atlas Climático do Rio Grande do Sul, elaborado por Matzenauer et al. (2011). O período considerado nos cálculos da evapotranspiração potencial foi de 1976 a 2005, sendo utilizada para a estimativa da

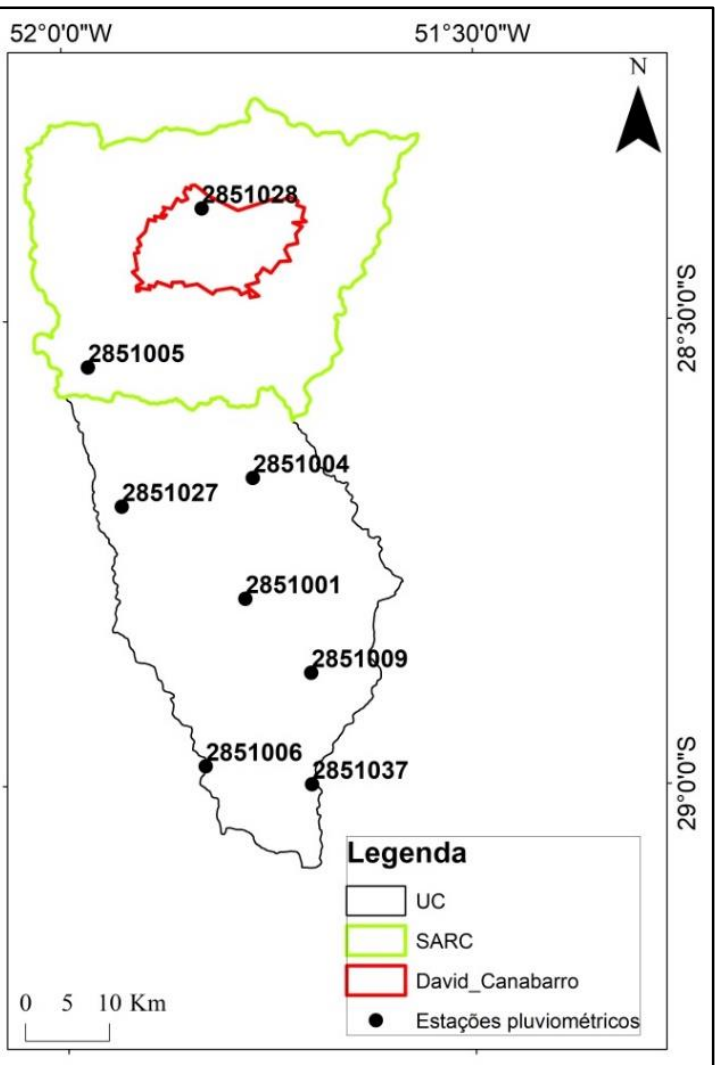

mesma o método de Thornthwaite (1948), para um intervalo mensal, sendo computado posteriormente, o valor anual. As estações climatológicas utilizadas estão apresentadas na figura 3 


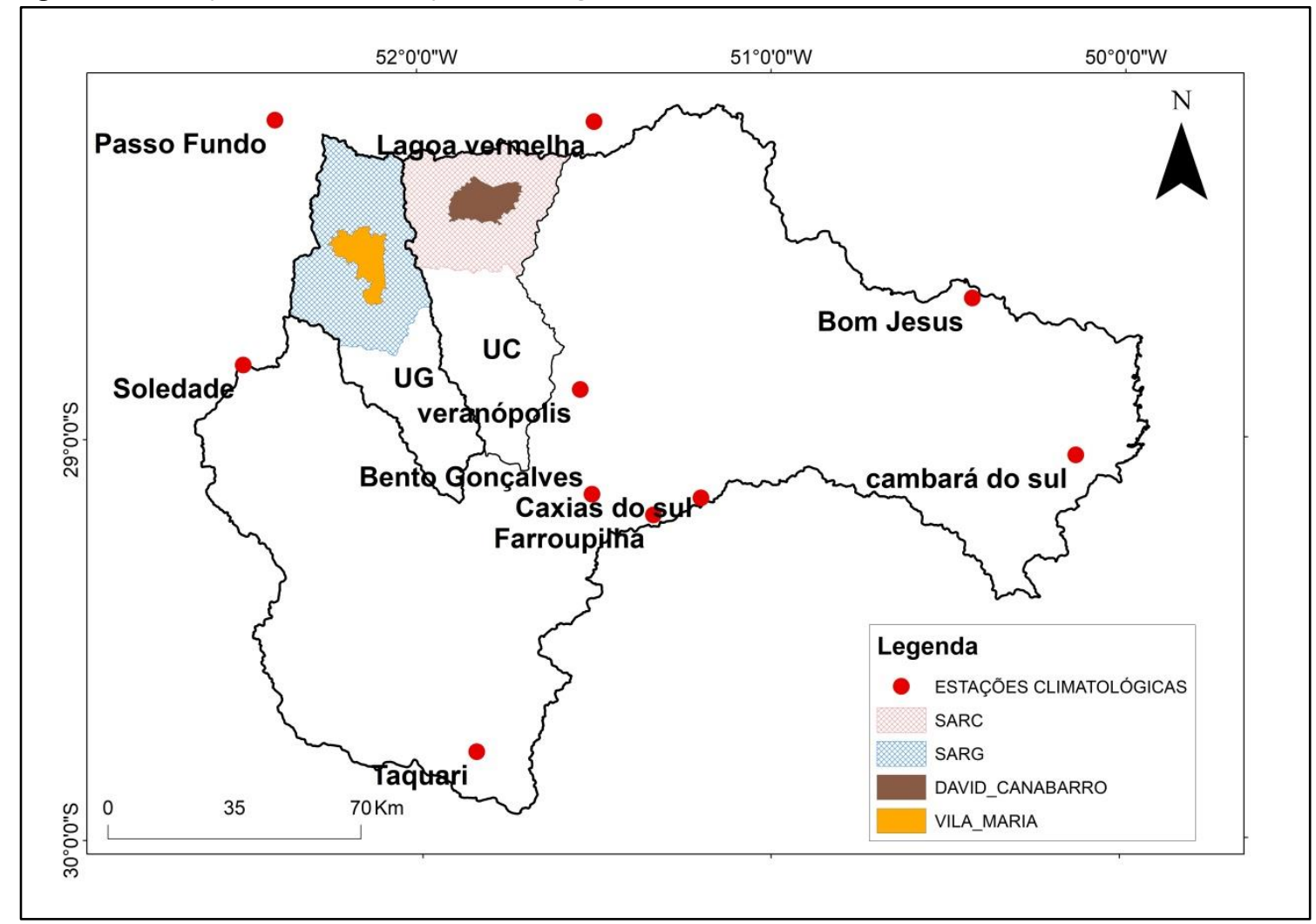

Como a evapotranspiração potencial (ETP), foi inferior a preci-

$C^{\prime}=\alpha . C$ pitação média anual em todas as áreas estudadas, a ETR foi considerada como sendo igual a ETP. Vale salientar, que conforme Tucci (2007), as diferenças entre as ETR e ETP reduzem sempre que os intervalos de tempo utilizados para o cálculo da segunda são ampliados, como no caso deste estudo que é anual.

O escoamento superficial foi calculado conforme equações 9 e 10:

Onde:

$\mathrm{C}=$ coeficiente de escoamento;

$\alpha=$ coeficiente que varia em função da declividade e estação seca ou úmida.

Os valores de coeficiente de escoamento superficial foram estimados conforme valores já tabelados, apresentados nos estudos de Carvalho (2013) adaptado de Fenn et al. (1975), conforme tabela 1. Os mesmos foram selecionados por se tratar $R_{\text {off }}=C^{\prime} . P$ de uma metodologia requerendo dados de fácil obtenção e rápida classificação.

Tabela 1 - Classificação do escoamento superficial.

\begin{tabular}{|c|c|c|c|}
\hline \multirow{2}{*}{ Tipo de solo } & \multirow{2}{*}{ Declividade (\%) } & \multicolumn{2}{|c|}{ Coeficiente $\alpha$} \\
\hline & & Estação seca & Estação úmida \\
\hline \multirow{3}{*}{ Arenoso $(C=0,35)$} & $<3$ & 0,43 & 0,65 \\
\hline & 3 a 7 & 0,54 & 0,7 \\
\hline & $>7$ & 0,6 & 0,7 \\
\hline \multirow{3}{*}{ Argiloso $(C=0,45)$} & $<3$ & 0,53 & 0,63 \\
\hline & 3 a 7 & 0,75 & 0,65 \\
\hline & $>7$ & 0,9 & 0,85 \\
\hline
\end{tabular}

Fonte: Carvalho (2013) 
Com base na variação mensal da precipitação, a estação seca é nos meses de junho a outubro e a úmida de novembro a maio (BORTOLIN, 2018). Para a classificação do tipo de solo, se arenoso ou argiloso, foi utilizado o mapa de solos do estado do Rio Grande do Sul na escala 1:1.000.000. Levantamentos mais detalhados de solos não foram desenvolvidos ainda na área de estudo, por isso a única fonte de informação existente é o mapa de solos utilizado. Contudo, vale salientar que estudos mais detalhados de solos terão influência nos resultados finais de estimativa da recarga.

O mapa de declividade foi elaborado no ArcGIS, a partir do modelo digital de elevação (MDE) da missão Shuttle Radar Topography Mission (SRTM), com resolução de 30 metros.

O potencial obtido através do estudo local e os potenciais obtidos a partir do valor de recarga de $8 \%$ previstos no Plano de Bacia (2011b) foram comparados com os três cenários de disponibilidades hídricas instaladas. A partir dessa comparação, foi realizada a análise do comprometimento dos recursos hí- dricos subterrâneos, consistindo na análise do potencial hídrico subterrâneo já utilizado através da disponibilidade hídrica instalada.

O nível de comprometimento do potencial hídrico foi definido com base na Deliberação CRH n 52/2005. 0 mesmo indica que quando o comprometimento do potencial é superior a $75 \%$, deve-se considerar com restrições os usos das águas subterrâneas. Baseado nisso, o nível de comprometimento do potencial hídrico foi classificado conforme apresentado na tabela 2 .

\section{RESULTADOS E DISCUSSÕES}

\subsection{Caracterização dos poços}

No banco de dados elaborado foram registradas as quantida des de poços apresentadas na tabela 3. A unidade de gestão, sub-bacia e município que apresentaram a maior quantidade de poços foram, respectivamente, a UC, SARG e Vila Maria.

Tabela 2 - Classificação do comprometimento do potencial hídrico subterrâneo

\begin{tabular}{ccc}
\hline \multicolumn{3}{c}{ Nível de comprometimento } \\
\hline $50 \%>$ (Dit/Po) & $50 \% \leq($ Dit $/ \mathrm{Po})<75 \%$ & $($ Dit $/ \mathrm{Po}) \geq 75 \%$ \\
Baixo & Médio & Crítico \\
\hline
\end{tabular}

Tabela 3 - Área e quantidade de poços inseridos em cada escala estudada

\begin{tabular}{cccc}
\hline Escalas & Quantidade total de poços & $\begin{array}{c}\mathrm{A} \\
\left(\mathrm{km}^{2}\right)\end{array}$ & Poço/ Km² \\
\hline UG & 816 & 2484,91 & 0,33 \\
UC & 1025 & 2561,44 & 0,40 \\
SARG & 521 & 1537,94 & 0,34 \\
SARC & 344 & 1247,64 & 0,28 \\
VM & 68 & 181,12 & 0,38 \\
DC & 50 & 175,37 & 0,29 \\
\hline
\end{tabular}

A distribuição espacial dos poços pode ser visualizada no mapa da figura 4, onde é possivel observar que a mesma ocorre de forma relativamente homogênea ao longo das áreas estudadas.
As informações presentes em cada área de estudo podem ser observadas na tabela 4. Com exceção das informações de tempo de bombeamento, todas as áreas estudadas possuem mais de $58 \%$ dos poços com as informações, contudo nenhum poço, nas áreas estudadas, possui todas as informações 
Figura 4 - Distribuição espacial dos poços nas áreas estudadas

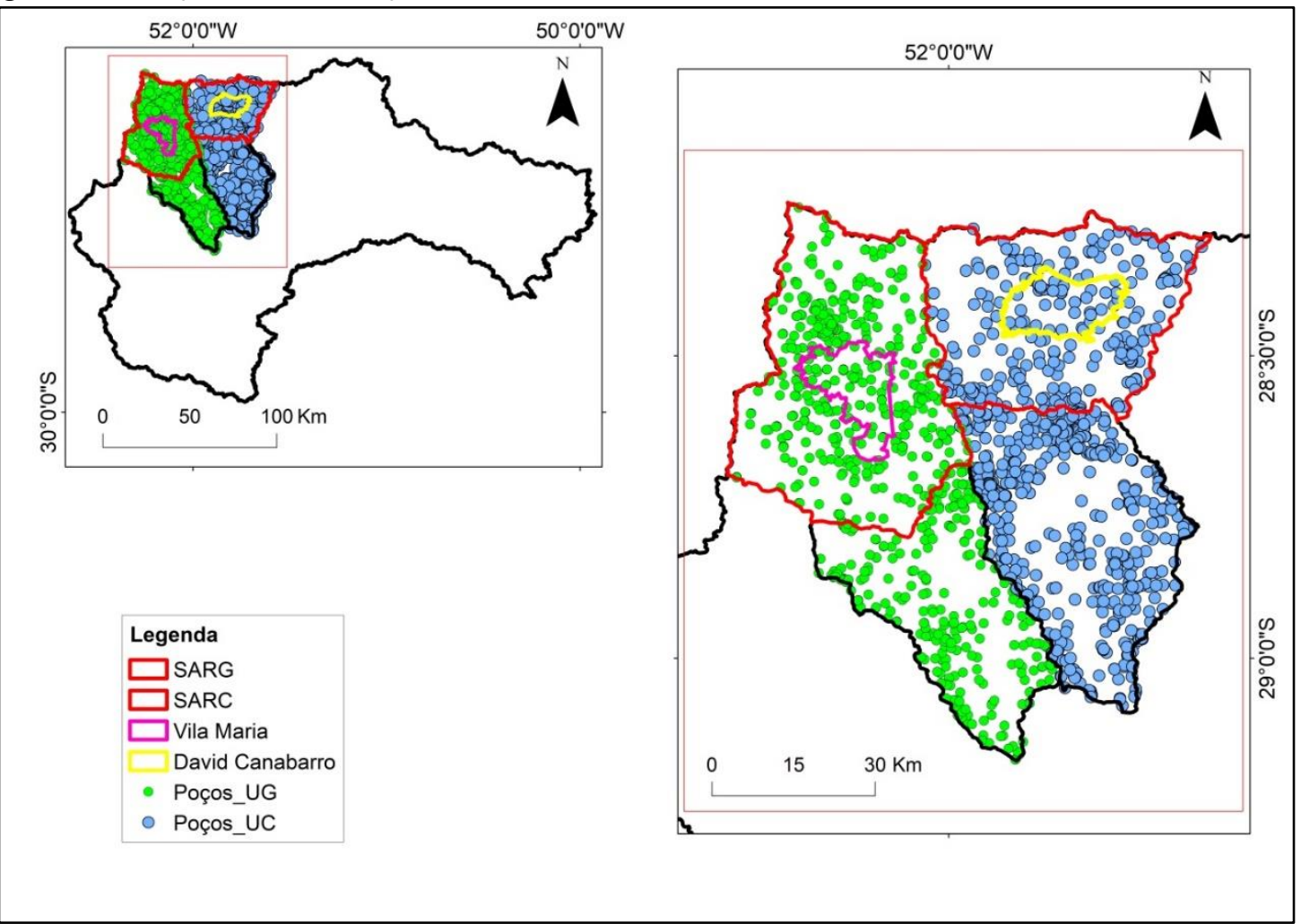

Tabela 4 - Quantidade de poços por informação

\begin{tabular}{ccccccc}
\hline \multirow{2}{*}{ Dados } & UG & UC & SARG & SARC & VM & DC \\
\hline C.g & $780(96 \%)$ & $907(88 \%)$ & $495(95 \%)$ & $297(86 \%)$ & $67(99 \%)$ & $46(92 \%)$ \\
Q & $720(88 \%)$ & $956(93 \%)$ & $511(98 \%)$ & $227(66 \%)$ & $51(75 \%)$ & $38(76 \%)$ \\
T.uso & $642(79 \%)$ & $599(58 \%)$ & $422(81 \%)$ & $212(62 \%)$ & $58(85 \%)$ & $33(66 \%)$ \\
T.b & $15(2 \%)$ & $72(7 \%)$ & $16(3 \%)$ & $17(5 \%)$ & 0 & 0 \\
T.i & 0 & 0 & 0 & 0 & 0 & 0 \\
\hline C.g: Coordenada Geográfica/ Q: Vazão/ T.uso: Tipo de uso da água/ T.b: Tempo de bombeamento/ T.i: Todas as informações
\end{tabular}

Os valores medianos de vazão são representativos do SASG, o qual possui vazões, em geral, baixas (REBOUÇAS \& FRAGA, 1988; REGINATO \& STRIEDER, 2006), conforme tabela 5. 0 maior valor mediano de vazão foi observado no município de David Canabarro (DC) e o menor no município de Vila Maria, respectivamente. Já os valores dos desvios padrões da vazão superaram os valores médios da mesma para a maioria das áreas analisadas, com exceção apenas do município de David Canabarro (Tabela 5). Logo, o valor mediano representa meIhor as vazões que ocorrem nas áreas estudadas.

Tabela 5 - Dados hidrodinâmicos dos poços

\begin{tabular}{ccccc}
\hline \multirow{2}{*}{ Escalas } & \multicolumn{3}{c}{$\mathrm{Q}\left(\mathrm{m}^{3} / \mathrm{h}\right)$} & tbp $(\mathrm{h})$ \\
& Mediana & Média & Desvio padrão & Média \\
\hline UG & 3,79 & 5,69 & 6,68 & 12,00 \\
SARG & 4,17 & 7,76 & 19,27 & 12,63 \\
VM & 2,50 & 6,15 & 8,60 & $\ldots .$. \\
UC & 4,12 & 7,85 & 10,57 & 12,04 \\
SARC & 5,00 & 10,15 & 16,55 & 12,24 \\
DC & 9,21 & 13,89 & 12,85 & $\ldots .$. \\
\hline
\end{tabular}


0 tempo de bombeamento projetado (tbp), em todas as áreas estudadas, apresentou valores muito próximos, não ultrapassando 13h. Nas escalas de município, que não há dados de tempo de bombeamento (tb), foi utilizado o valor médio calculado na sub-bacia em que o mesmo está inserido. Logo, o tempo de bombeamento dos poços em David Canabarro foi de 12,63h e em Vila Maria, de 12,24h.

\subsection{Disponibilidade hídrica instalada total (Dit)}

Entre as unidades de gestão UC e UG foi observado uma diferença significativa dos valores de Dit (Tabela 6). Isso ocorreu pelo maior número de poços existente na UC e pela vazão mediana e tempo de bombeamento médio serem superiores nesta unidade também.
Nas sub-bacias ocorreram valores aproximados de Dit, no entanto, a SARC corresponde a 35\% da Dit da unidade de gestão UC, onde a mesma está inserida e a SARG corresponde a 79\% da Dit da unidade de gestão UG.

Mesmo com uma quantidade de poços maior que a do município de David Canabarro, o município de Vila Maria apresentou disponibilidade hídrica inferior ao mesmo. Isso ocorreu devido à vazão mediana do município de David Canabarro ser significativamente superior a do município de Vila Maria. 0 município de David Canabarro abrange cerca de 16\% da Dit da sub-bacia SARC, onde o mesmo está situado, já o município de Vila Maria compreende apenas $8 \%$ da Dit da sub-bacia SARG.

Tabela 6 - Disponibilidades hídricas instaladas

\begin{tabular}{|c|c|c|c|c|c|c|}
\hline \multirow[b]{2}{*}{ Escalas } & \multicolumn{3}{|c|}{ Dit (hm³/ano) } & \multicolumn{3}{|c|}{ Dit1,5 (hm³/ano) } \\
\hline & $\begin{array}{c}\text { Tbp } \\
\text { (h) }\end{array}$ & $\begin{array}{c}\mathrm{tb} \\
24 \mathrm{~h}\end{array}$ & $\begin{array}{c}\mathrm{tb} \\
18 \mathrm{~h}\end{array}$ & $\begin{array}{l}\text { Tbp } \\
\text { (h) }\end{array}$ & $\begin{array}{c}\text { tb } \\
18 \mathrm{~h}\end{array}$ & $\begin{array}{c}\text { tb } \\
24 h\end{array}$ \\
\hline UC & 43,94 & 65,37 & 87,15 & 65,90 & 98,05 & 130,73 \\
\hline SARC & 15,46 & 22,49 & 29,98 & 23,18 & 33,73 & 44,97 \\
\hline David Canabarro & 2,54 & 4,98 & 3,74 & 3,81 & 5,61 & 7,47 \\
\hline UG & 24,55 & 36,78 & 49,04 & 36,83 & 55,17 & 73,56 \\
\hline SARG & 19,30 & 27,30 & 36,43 & 28,95 & 40,94 & 54,64 \\
\hline Vila Maria & 1,66 & 2,36 & 3,15 & 2,49 & 3,54 & 4,73 \\
\hline
\end{tabular}

Na tabela 6, também é possível observar os valores de Dit calculados para os tempos de bombeamento projetado, de $18 \mathrm{e}$ 24 horas acrescidos em 50\%, sendo que esses foram os resultados de Dit utilizados posteriormente para os cálculos de comprometimento do potencial hídrico subterrâneo.

\subsection{Recarga}

Os valores de todas as variáveis envolvidas na estimativa da recarga (precipitação, evapotranspiração, escoamento superficial), apresentaram pouca variação entre si (Tabela 7). Isso se deve a proximidade das unidades de gestão, as quais compartilham 3 estações pluviométricas (2851005, 2851027 e 2851006), e ao fato de que todas as áreas estudadas possuem predominantemente solos argilosos com declividades acima de $7 \%$.

Tabela 7 - Precipitação, evapotranspiração, escoamento superficial e recarga média anual de cada área estudada.

\begin{tabular}{cccccc}
\hline Escalas & $\begin{array}{c}\text { P } \\
(\mathrm{mm} / \mathrm{ano})\end{array}$ & $\begin{array}{c}\text { EVP } \\
(\mathrm{mm} / \mathrm{ano})\end{array}$ & $\begin{array}{c}\text { ESC } \\
(\mathrm{mm} / \mathrm{ano})\end{array}$ & $\mathrm{R}$ (mm/ano) & $\% \mathrm{P}$ \\
\hline UG & 1637,59 & 832,5 & 559,15 & 245,94 & $15,02 \%$ \\
UC & 1602,22 & 815,0 & 562,65 & 224,57 & $14,02 \%$ \\
SARG & 1629,68 & 817,5 & 581,12 & 231,06 & $14,18 \%$ \\
SARC & 1647,95 & 800,0 & 567,32 & 280,63 & $17,03 \%$ \\
Vila Maria & 1595,48 & 815,0 & 586,31 & 194,17 & $12,17 \%$ \\
David Canabarro & 1658,08 & 802,5 & 602,01 & 253,57 & $15,29 \%$ \\
\hline
\end{tabular}

Os valores dos parâmetros apresentados na tabela 7 foram condizentes com os valores encontrados por outros autores em estudo na bacia Taquari-Antas, conforme apresentado a seguir.

A precipitação média anual nas áreas estudadas variou de 1595,48 a $1658,08 \mathrm{~mm}$, conforme apresentado na tabela 7 .
Os valores de precipitação estimados foram semelhantes aos observados na bacia Taquari-Antas por Collischonn (2001), de 1600 a 1800 mm; por Rossato (2011), entre 1290 e 2000 mm; e por Capalonga (2017), de 1573 a $1897 \mathrm{~mm}$.

Os valores médios de evapotranspiração variaram de 800 a $832 \mathrm{~mm} / \mathrm{ano}$ nas áreas estudadas. Esses valores foram se- 
melhantes aos observados, para a bacia, por Capalonga (2017), de 760 mm, e por Collischonn e Tucci (2001), que estimaram o valor médio de $872 \mathrm{~mm}$.

O escoamento superficial em todas as escalas foi semelhante, sendo o menor valor estimado de 559,15 mm (na unidade de gestão UC) e o maior de 602,01 mm (no município de David Canabarro), conforme apresentado na tabela 7 . Os valores de escoamento observados na tabela 7 , em sua maioria, se encontram dentro do intervalo predominante de escoamento de 501 a 600 mm/ano, estimado por Capalonga (2017) para bacia Taquari-Antas, e abaixo do valor observado por Collischonne Tucci (2001) de 768 mm/ano.

A recarga nas unidades de gestão, sub-bacias e municípios também estão apresentadas na tabela 7 , onde é possível observar que ocorreram valores de recarga muito semelhantes entre as unidades de gestão UC e UG, a Sub-bacia SARG e o município de David Canabarro, todos com recargas em torno de $15 \%$ da precipitação média anual. As maiores recargas foram observadas na Sub-bacia SARC e o menor valor de recarga reside no município de Vila Maria, sendo a mesmo de $12,17 \%$ da precipitação, contudo, essa recarga está acima do valor previsto no Plano de Bacia (2011b), de $8 \%$ da precipitação média anual. Com exceção da recarga do município de Vila Maria, as demais áreas estudadas apresentaram valores próximos ao valor médio de 293 mm/ano estimado por Capalonga (2017) para a bacia Taquari-Antas. Os valores também ficaram próximos aos observados por Bortolin et al. (2016), de $18 \%$ da precipitação média anual.

\subsection{Potencial hídrico subterrâneo (Po)}

Os valores de potenciais hídricos subterrâneos, estimados através de análises locais nas áreas de estudo selecionadas e dos dados de recarga calculados no Plano de Bacia (2011b), estão apresentados no gráfico da figura 5. Em todas as áreas estudadas, os valores de potencial previstos a partir do estudo local foram superiores aos obtidos através da recarga estimada feita com base nos dados do plano da bacia TaquariAntas. Em média, os valores de potencial obtidos com os dados da bacia foram $45 \%$ inferiores aos estimados através dos estudos locais, sendo que a maior diferença foi observada na sub-bacia SARC, que foi 53,02\% inferior. Isso ocorreu porque, nos estudos locais das diferentes áreas, foram estimados valores de recarga superiores a $8 \%$.

Figura 5 - Comparação entre o potencial hídrico subterrâneo estimado com os dados do plano e o potencial estimado a partir de estudos locais

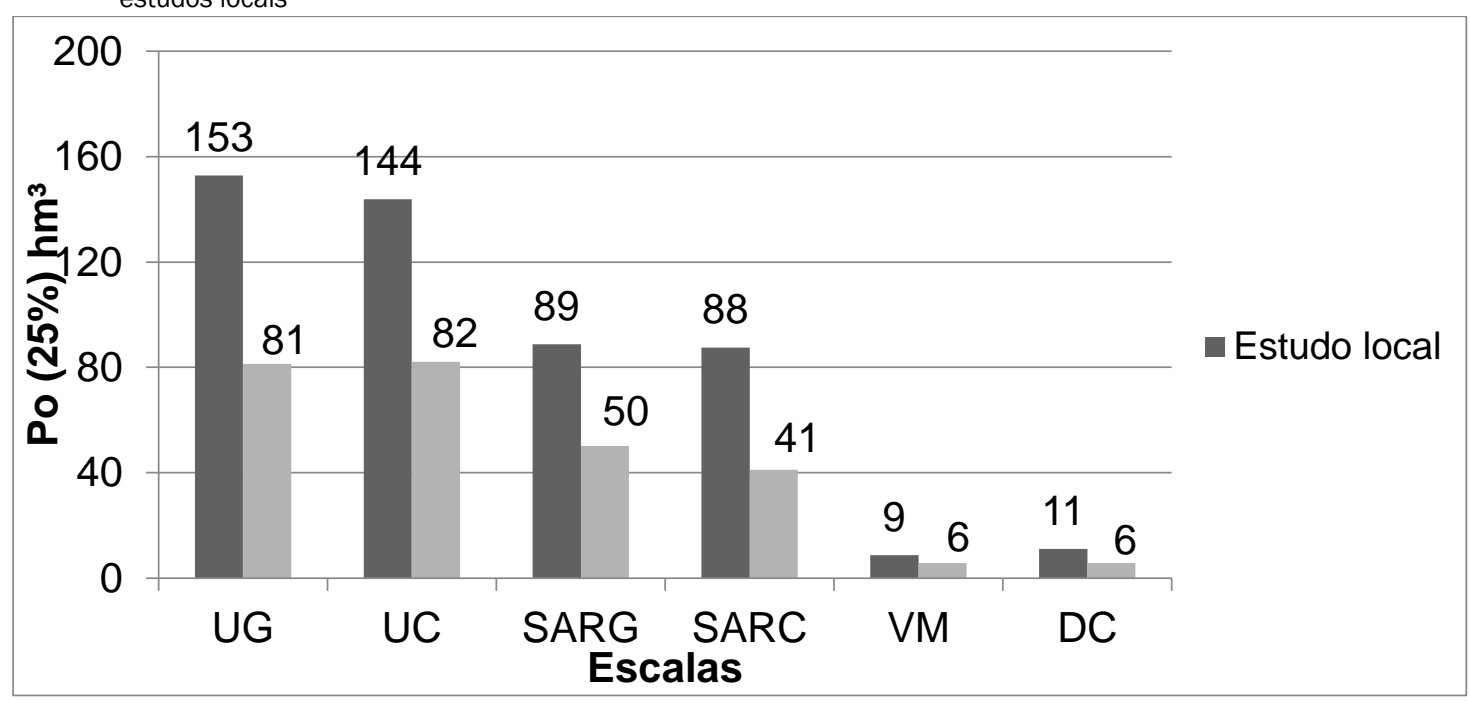

Os estudos mais detalhados em cada área de estudo selecionada originaram um volume maior de água disponível para ser utilizada, o que é, para as regiões estudas, de suma importância frente à significativa utilização dos recursos hídricos subterrâneos para o desenvolvimento social e econômico dessas regiões. Desta forma, para regiões com características semeInantes a da bacia Taquari-Antas se fazem necessários estudos em maior detalhe, para que a gestão dos mesmos ocorra de forma coerente com a realidade do potencial hídrico subterrâneo de cada região.

No Plano de Bacia (2011b), não foi indicada a metodologia utilizada para a estimativa da recarga. Diferentes metodologias podem originar diferentes volumes de recarga, e uma análise com a aplicação de diferentes métodos para a estimativa da recarga seria interessante para se ter valores mais precisos da mesma. Bortolin (2018), ao analisar duas sub-bacias na Bacia Taquari-Antas, através de 4 variações de métodos de balanço hídrico, 12 métodos baseados em águas superficiais, 2 modelos de simulação de bacia, técnicas de regressão múltipla e o método de variação dos níveis de água com diferentes abordagens, resultou em estimativas de recarga que variaram entre 12 e 55\% da precipitação da região. A importância da diferença observada entre as duas estimativas, fica melhor descrita ao se fazer a análise do comprometimento do potencial hídrico subterrâneo, conforme apresentado no item a seguir.

\subsection{Análise do comprometimento do potencial hídrico sub- terrâneo}

O comprometimento do potencial hídrico subterrâneo, reali- 
zado com os estudos locais, apresentou nível de comprometimento baixo para todas as áreas estudadas apenas para o cenário de Dittbp, conforme apresentado na figura 6 . 0 comprometimento estimado comparando o potencial com a Dit ${ }_{18 \mathrm{~h}}$, apresentou níveis baixos de comprometimento na maior parte das áreas analisadas, com exceção do município de David Canabarro e da unidade de gestão UC, os quais apresentaram nível de comprometimento médio. Para o cenário mais crítico de comprometimento, estimado através da Dit $24 \mathrm{~h}$, foram observados níveis de comprometimento médio em mais de uma área, sendo elas: os municípios de Vila Maria e David Canabarro e as sub-bacias SARC e SARG. Para esse cenário foi calculado o nível de comprometimento crítico apenas para a unidade de gestão UC.

Figura 6 - Mapa de comprometimento desenvolvido através da análise em estudo local. 1: análise do comprometimento realizada através da Di calculada com tbp. 2: análise do comprometimento realizada através da Di calculada com tb (18h). 3: análise do comprometimento realizada através da Di calculada com tb (24h)

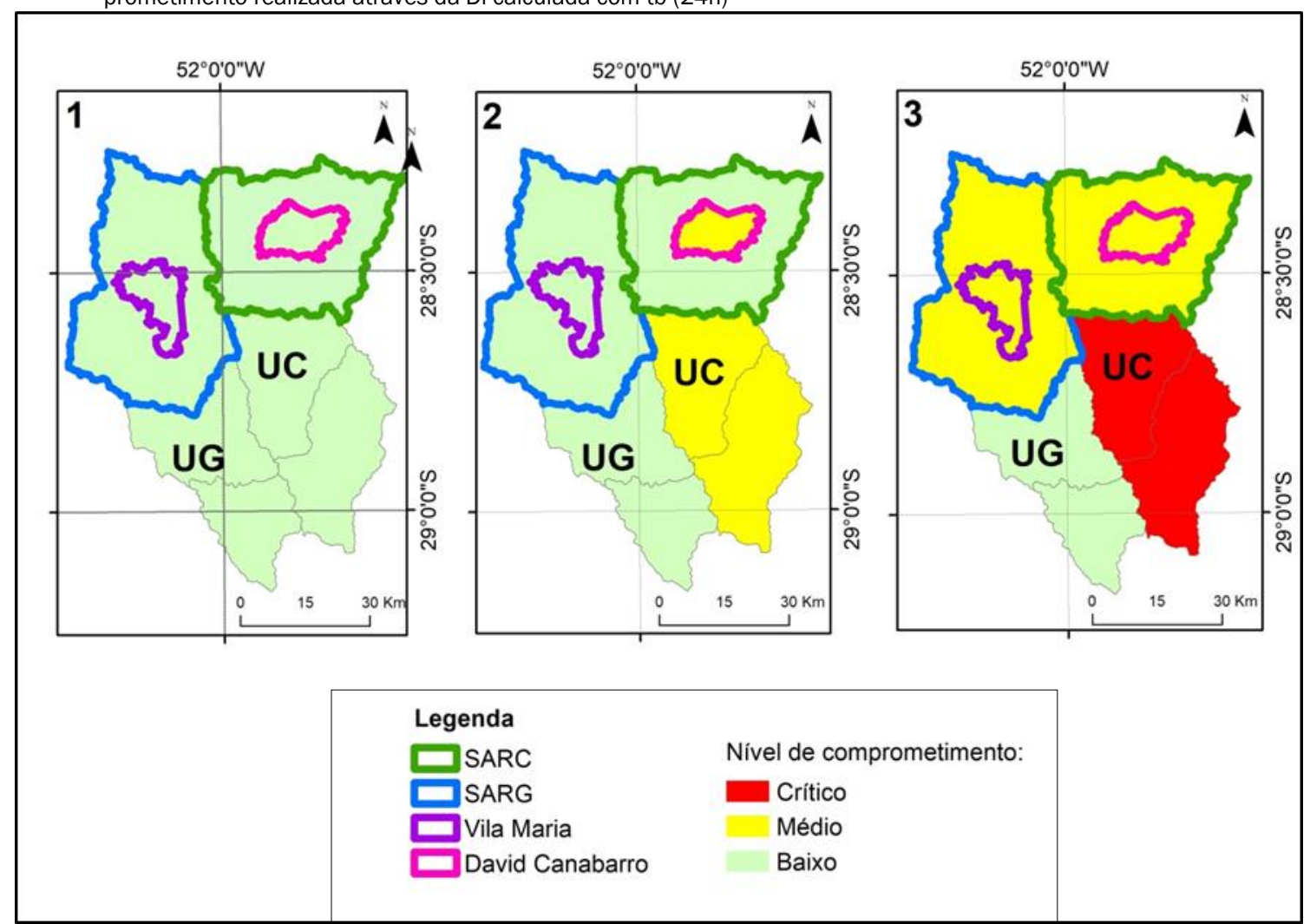

Conforme pode ser observado na figura 6 e apresentado na tabela 8, o potencial hídrico subterrâneo nos três cenários simulados, estimado através de estudos locais, apresentaram volumes de Dit inferior ao potencial hídrico subterrâneo calculado. Por meio dos estudos locais, a unidade de gestão UC se demonstrou a área que necessita de maior atenção, sendo a única a apresentar nível de comprometimento crítico para um dos cenários simulados. Além disso, as unidades que a compõem só apresentaram nível de comprometimento baixo para o cenário de Dittbp, sendo este o menos crítico.

Tabela 8 - \% do comprometimento do potencial hídrico subterrâneo estimado com os estudos locais

\begin{tabular}{cccccccc}
\hline \multirow{2}{*}{ Escalas } & $\begin{array}{c}\text { Po25\% } \\
\left.\text { (hm }^{3}\right)\end{array}$ & tbp (h) & tb 18(h) & tb 24(h) & tbp (h) & tb 18(h) & tb 24(h) \\
\hline UG & 152,78 & 36,83 & 55,17 & 73,56 & $24 \%$ & $36 \%$ & $48 \%$ \\
UC & 143,81 & 65,90 & 98,05 & 130,73 & $46 \%$ & $68 \%$ & $91 \%$ \\
SARG & 88,84 & 28,95 & 40,94 & 54,64 & $33 \%$ & $46 \%$ & $62 \%$ \\
SARC & 87,53 & 23,18 & 33,73 & 44,97 & $26 \%$ & $39 \%$ & $51 \%$ \\
VM & 8,79 & 2,49 & 3,54 & 4,73 & $28 \%$ & $40 \%$ & $54 \%$ \\
DC & 11,12 & 3,81 & 7,47 & 5,61 & $34 \%$ & $50 \%$ & $67 \%$ \\
\hline
\end{tabular}


Como o Plano de Bacia (2011b) estimou uma recarga menor que a calculada a partir dos estudos locais, o nível de comprometimento do potencial hídrico subterrâneo foi maior ao observado anteriormente com base nos estudos locais (Figura 7). Somente a unidade de gestão UG apresentou nível baixo de comprometimento do potencial e para o cenário menos crítico de Dittbp. Para os demais cenários e áreas estudadas, o nível de comprometimento foi de médio a crítico, atingindo no cenário de Dit24h o nível crítico em todas as áreas avaliadas.

Figura 7 - Mapa de comprometimento desenvolvido através da recarga estimada no plano de bacia. 1: análise do comprometimento realizada através da Di calculada com tbp. 2: análise do comprometimento realizada através da Di calculada com tb (18h). 3: análise do comprometimento realizada através da Di calculada com tb $(24 \mathrm{~h})$

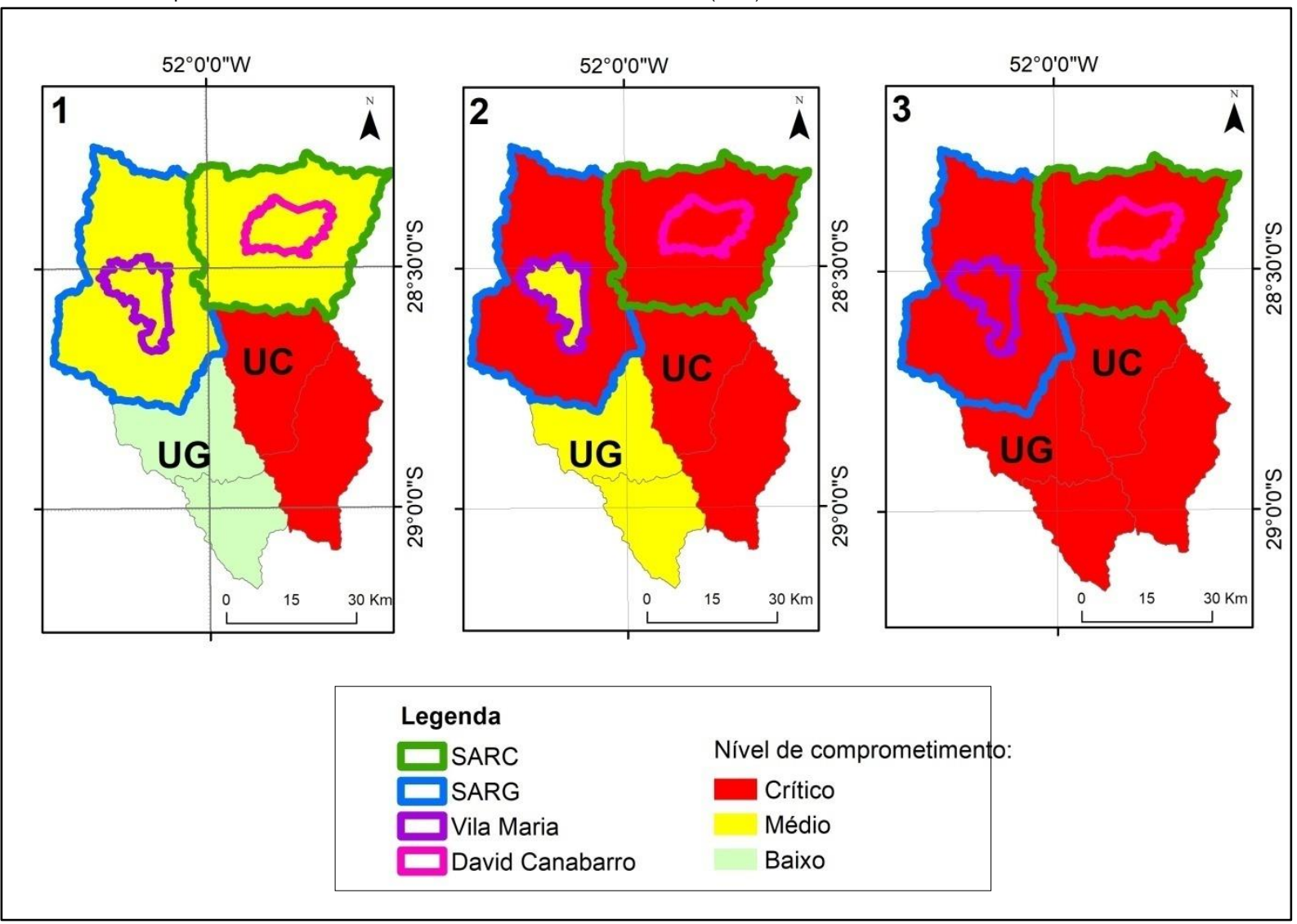

Ao contrário do observado nos estudos locais, a Dit superou os valores de potencial calculados através da recarga prevista no Plano de Bacia (2011b). Na unidade de gestão UC, a Dit ${ }_{18 \mathrm{~h}}$ ultrapassou quase $20 \%$ do potencial estimado para a área, enquanto a Dit24h ultrapassou cerca de $60 \%$ desse mesmo potencial, conforme apresentado na tabela 9. Assim como observado através dos estudos locais, a unidade de gestão UC é a que exige maior atenção; sendo que, as demais áreas que apresentaram valores de Dit acima do potencial ocorreram somente no cenário mais crítico, de Dit24h. Dessas regiões, a área que se destaca é o município de David Canabarro, com quase $30 \%$ de Dit acima do potencial estimado para a região.

Tabela 9 - \% do comprometimento do potencial hídrico subterrâneo estimado com os 8\% de recarga do Plano de Bacia (2011b)

\begin{tabular}{cccccccc}
\hline \multirow{2}{*}{ Escalas } & \multirow{2}{*}{ Pop 8\% $\left(\mathrm{hm}^{3}\right)$} & \multicolumn{3}{c}{ Dit(hm $\mathbf{3} / \mathrm{ano})$} & \multicolumn{3}{c}{ Comprometimento de Po } \\
& & tbp (h) & tb 18(h) & tb 24(h) & tbp (h) & tb 18(h) & tb 24(h) \\
\hline UG & 81,39 & 36,83 & 55,17 & 73,56 & $45 \%$ & $68 \%$ & $90 \%$ \\
UC & 82,08 & 65,9 & 98,05 & 130,73 & $80 \%$ & $119 \%$ & $159 \%$ \\
SARG & 50,13 & 28,95 & 40,94 & 54,64 & $58 \%$ & $82 \%$ & $109 \%$ \\
SARC & 41,12 & 23,18 & 33,73 & 44,97 & $56 \%$ & $82 \%$ & $109 \%$ \\
VM & 5,78 & 2,49 & 3,54 & 4,73 & $43 \%$ & $61 \%$ & $82 \%$ \\
DC & 5,82 & 3,81 & 5,61 & 7,47 & $65 \%$ & $96 \%$ & $129 \%$ \\
\hline
\end{tabular}


Os resultados oriundos dos estudos locais indicaram um panorama menos crítico do comprometimento do potencial hídrico existente em cada área analisada, se comparados aos niveis de comprometimento observados através do potencial calculado com a recarga prevista no Plano de Bacia (2011b). Tanto a análise realizada em escala local quanto a efetuada com os dados do Plano de Bacia (2011b) demostraram uma situação preocupante do nível do comprometimento do potencial hídrico subterrâneo na unidade de gestão UC. Contudo, o objetivo desta análise é demostrar a importância de um estudo de maior detalhe para a estimativa do potencial hídrico subterrâneo, e não deve servir de base para a realização de restrições de novas perfurações na unidade de gestão em questão, mas sim indicar a necessidade de uma maior atenção dos órgãos gestores para a mesma. Faz-se necessários para tais constatações, estudos mais aprofundados e com diferentes métodos de avaliação da variação espacial e temporal da recarga na região.

A utilização de dados do Plano de Bacia (2011b), aplicados na avaliação do potencial hídrico subterrâneo de unidades menores, como municípios, sub-bacias e unidades de gestão, resultou em valores de potencial hídrico subterrâneo inferiores aos estimados localmente em cada região.

Se forem considerados os dados do Plano de Bacia (2011b), para a realização da gestão do aquífero, a mesma seria realizada de forma mais conservadora do que o necessário. As áreas que foram analisadas são predominantemente abastecidas por água subterrânea, sendo os municípios supridos exclusivamente pela mesma. Logo, qualquer variação no potencial hídrico subterrâneo pode e irá influenciar o desenvolvimento econômico e social da região, sendo os planos de bacia uma das base de informação para os processos de outorgas. Além disso, as áreas estudadas estão inseridas no SASG, o qual possuí características anisotrópicas e heterogênias, as quais demandam estudos detalhados para a sua correta gestão e utilização.

\section{CONCLUSÕES}

Com o estudo desenvolvido ficou evidente que há uma diferença entre o potencial hídrico obtido através de estudos locais e o potencial obtido aplicando o valor de recarga estimado no Plano de Bacia (2011b).

Em média, o valor de potencial obtido com os dados da bacia foi $45 \%$ inferior ao estimado através dos estudos locais, devido principalmente às diferentes recargas subterrâneas calculadas nesse estudo e às disponíveis no plano de bacia. Tal diferença refletiu no nível de comprometimento da água subterrânea nas áreas estudadas, o qual variou de médio a crítico para todos os cenários de disponibilidade hídrica instalada, com exceção apenas da unidade de gestão UG, onde o nível de comprometimento foi baixo para o cenário de Dittbp. A partir dos estudos locais foi observado um nível de comprometimento crítico apenas na unidade de gestão UC, no cenário de Dit24h.

Logo, a realização de estudos mais detalhados de potencial hídrico subterrâneo permitirá o gerenciamento mais coerente do mesmo, adotando cenários de maior ou menor restrição levando em consideração o nível de conhecimento hidrogeológico e da disponibilidade hídrica instalada existente na bacia. Para regiões que possuem apenas a água subterrânea como fonte de recursos hídricos, qualquer variação no potencial pode significar avanços ou retrocessos na economia e na qualidade de vida da população. Logo, esta pesquisa serve como alerta para que nos planos de bacias sejam desenvolvidos estudos mais detalhados de recarga, para melhor estimativa dos potenciais hídricos subterrâneos existentes.

Vale salientar que estudos realizados em escalas mais detaIhadas não excluem a necessidade de uma gestão integrada dos recursos hídricos, observando as ações realizadas na bacia como um todo, as quais poderão afetar as áreas inseridas na mesma, como unidades de gestão, sub-bacias e municípios.

\section{REFERÊNCIAS}

BORTOLIN, T.A. Estudo da recarga do Sistema Aquífero Serra Geral na Bacia Hidrográfica Taquari-Antas. Tese (Doutorado) Instituto de Pesquisas Hidráulicas da UFGRS, Porto Alegre, 2018. 220p.

BORTOLIN, T.A; REGINATO, P.A.P; SCHNEIDER, V.E. 2016. Estimativa de recarga pelo método do balanço hídrico em uma bacia hidrográfica com ocorrência de aquíferos fraturados. In: CONGRESSO BRASILEIRO DE ÁGUAS SUBTERRÂNEAS, 19., 2016. Anais... Campinas-São Paulo.

BRASIL. Lei n. 9.433, de 8 de jan. de 1997. Institui a Política Nacional de Recursos Hídricos, cria o Sistema Nacional de Gerenciamento de Recursos Hídricos. Brasília, DF, jan 1997.

CAPALONGA, S. Avaliação do risco de poluição da água subterrânea da bacia hidrográfica do rio Taquari-Antas. Trabalho de conclusão de curso (Graduação) - Escola de engenharia, Instituto de Pesquisas Hidráulicas, Universidade Federal do Rio Grande do Sul, 2017.

CARVALHO, A. M. Modelagem numérica como ferramenta para a gestão das águas subterrâneas em São José do Rio Preto, SP. Dissertação (Mestrado) - Instituto de Geociências da Universidade de São Paulo, São Paulo, 2013.

CENSO DEMOGRÁFICO 2010. Características da população e dos domicílios: resultados do universo. Rio de Janeiro: IBGE, 2011.

COLLISCHONN, W; TUCCI, C.E.M. Simulação hidrológica de grandes bacias. Revista Brasileira de Recursos Hídricos, v. 6, n.1, p. 95-118, Jan./Mar. 2001.

COLLISCHONN, W. Simulação hidrológica de grandes bacias. Tese (Doutorado) - Instituto de Pesquisas Hidráulicas da UFGRS, Porto Alegre, 2001. 270p.

COSTA, W. D. Avaliação das reservas, potencialidades e disponibilidade de aqüífero. In: CONGRESSO BRASILEIRO DE ÁGUAS SUBTERRÂNEAS, 10., 1998. Anais.... São Paulo: ABAS. 1998. p. Trabalho 50, p 11.

FENN,D. HANLEY, K., DE GEARE, T. Use of the Water Balance Method for predicting leachate generation from solid waste disposal sites. US Environmental Protection Agency Report. 1975.

HEALY, R.W.; COOK, P.G. Using groundwater levels to estimate 
recharge. Hydrogeology Journal. v.10, n. 1. p. 91-109, 2002.

MACHADO, J.L.F.; FREITAS, M.A. de. Projeto Mapa Hidrogeológico do Rio Grande do Sul: relatório final. Porto Alegre. CPRM. 65p. il. mapa. 2005.

MATZENAUER, R.; RADIN, B.; ALMEIDA, I.R.(E d.). Atlas Climático: Rio Grande do Sul. Porto Alegre: Secretaria da Agricultura Pecuária e Agronegócio; Fundação Estadual de Pesquisa Agropecuária - FEPAGRO, 2011.

PLANO DE BACIA A. Elaboração de serviços de consultoria relativa às fases $A$ e $B$ do Plano de Bacia Taquari Antas. Relatório Técnico 01. STE- Serviços Técnicos de Engenharia S.A. 2011.

PLANO DE BACIA B. Elaboração de serviços de consultoria relativa às fases $A$ e $B$ do Plano de Bacia Taquari Antas: Fase $A$ - Diagnóstico e Prognóstico (Etapa A.1 a A.2). Relatório Técnico 02 (TOMO I). STE- Serviços Técnicos de Engenharia S.A. 2011.

PLANO DE BACIA C. Elaboração de serviços de consultoria relativa às fases $A$ e $B$ do Plano de Bacia Taquari Antas: Fase $A$ - Diagnóstico e Prognóstico (Etapa A.1 a A.2). Caderno Temático: Saneamento Básico. STE- Serviços Técnicos de Engenharia S.A. 2011.

REBOUÇAS, A. da C.; FRAGA, C. G. Hidrogeologia das Rochas Vulcânicas do Brasil. Revista Águas Subterrâneas, v. 12, n. 1, p. 30-55, 1988.
REGINATO, P.A.R.; STRIEDER, A.J. Caracterização Hidrogeológica e Potencialidades dos Aqüíferos Fraturados da Formação Serra Geral na Região Nordeste do Estado do Rio Grande do Sul. In: CONGRESSO BRASILEIRO DE ÁGUAS SUBTERRÂNEAS, 13., 2004. Anais... Cuiabá, Anais (CdRoom). 2004.

REGINATO, P.A.R.; STRIEDER, A.J. Integração de Dados Geológicos na Prospecção de Aquíferos Fraturados na Formação Serra Geral. Revista da Associação Brasileira de Águas Subterrâneas, v. 20, n.1, p. 1-14, 2006.

ROSSATO. Os climas do Rio Grande do Sul: variabilidade, tendências e tipologia. Tese (Doutorado) - Universidade Federal do Rio Grande do Sul. Instituto de Geociências. Programa de Pós-Graduação em Geografia. 2011.

THORNTHWAITE, C. W. An approach toward a rationalclassificationofclimate. Geograph.Rev., 38, 55-94, 1948.

TODD, D. Groundwater hydrology. New York: John Wiley \& Sons, 1959. 319p.

TUCCI, C.E.M. Hidrologia: ciência e aplicação. 4.ed. Porto Alegre: Ed. da UFRGS, 2007. p.943.

VANCONCELOS, S.M.S. Avaliação da Recarga Subterrânea Através da Variação do Nivel Potenciométrico no Aqüífero Dunas/Paleodunas, Fortaleza, Ceará . RBRH - Revista Brasileira de Recursos Hídricos, v. 10. n.2. p. 49-57. 2005. 